BABELAO 2 (2013), p. 87-104

(C) ABELAO (Belgium)

\title{
Les lieux de rédaction des lettres de Paul d'après la tradition manuscrite
}

par

Christian-B. AMPHOUX

CPAF, Université d'Aix-Marseille

L es épittres du corpus paulinien ont été rassemblées au cours $\mathrm{du} 2^{\mathrm{e}} \mathrm{s}$. pour former une collection de quatorze épîtres au premières éditons, nous nes nous sont parvenues à travers ces premieres éditions, hous ne connaissons pas directement le texte écrit (ou dicté) par l'apôtre Paul et nous n'avons que peu d'indices externes pour distinguer celles qui sont de lui et celles qui ont été écrites après lui. Dans la tradition manuscrite, une subscriptio, "souscription", est ajoutée à la fin de chaque lettre, attestée à partir du $5^{\mathrm{e}}$ s., mentionnant non seulement les destinataires de la lettre qui s'achève et ceux de la lettre suivante, mais aussi, entre ces deux données, le lieu présumé de la rédaction et parfois le nom du ou des copistes qui ont écrit sous la dictée. Contrairement aux évangiles, aucune indication de date n'est fournie. Les souscriptions figurent avec leurs témoins dans l'apparat critique de C. Tischendorf ${ }^{1}$, elles sont reprises dans le Nestle ${ }^{2}$; et pour le

${ }^{1}$ C. TisCHENDORF, Novum Testamentum graece, ed. $8^{\text {a }}$ critica maior, vol. 2 , Leipzig, 1872. 
latin, dans l'édition critique de Wordworth et White ${ }^{3}$. Nous n'avons pas consulté les données d'autres langues. On pourrait d'ailleurs douter de l'intérêt des souscriptions, introduites tardivement; mais à l'examen, on constate que certaines indications de lieu semblent fiables et d'autres, non: les souscriptions ne sont pas à ignorer, mais elles demandent un examen critique. Pour certaines lettres, plusieurs lieux sont en concurrence: peut-on déterminer quel est le lieu de rédaction le plus probable de chaque lettre et rendre compte des autres lieux proposés?

\section{Les lieux indiqués dans les souscriptions}

Tous les manuscrits ne présentent pas d'indications de lieu de rédaction; et quand c'est le cas, pour les manuscrits les plus anciens, elles ont été ajoutées après la copie: c'est le cas pour le Vaticanus (B.03) et le Claromontanus (D.06), deux des plus anciens manuscrits à présenter des indications de lieu, et nous l'indiquons en ajoutant à la lettre qui les désigne un exposant $\left(B^{1}\right.$, $\left.\mathrm{D}^{1}\right)$, comme le fait le Nestle.

Voici d'abord les attestations de lieux de rédaction données dans les souscriptions.

\begin{tabular}{|c|c|c|}
\hline Romains & 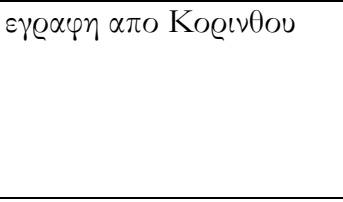 & 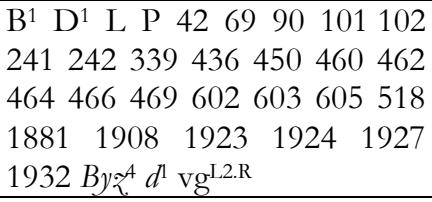 \\
\hline \multirow[t]{2}{*}{1 Corinthiens } & 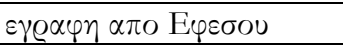 & $\mathrm{B}^{1} \mathrm{P} 462945 p c$; Euthalius ${ }^{5}$ \\
\hline & $\varepsilon \gamma \varrho \alpha \varphi \eta \alpha \pi O \Phi_{i} \lambda \iota \pi \pi \omega \nu$ & 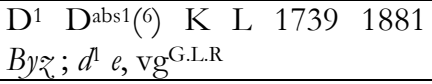 \\
\hline \multirow[t]{3}{*}{2 Corinthiens } & $\varepsilon \gamma \varrho \alpha \varphi \eta \alpha \pi O \Phi_{i} \lambda \iota \pi \pi \omega \nu$ & $\begin{array}{l}\text { B1 K L P } 81104205209328 \\
33717391881 \text { Byz; d } \text { vg }^{\mathrm{L}}\end{array}$ \\
\hline & écrite de Troas & $\operatorname{vg}^{\mathrm{R}}$ \\
\hline & écrite de Macédoine & $\mathrm{vg}^{\text {H.T.U. } \Theta}$ \\
\hline \multirow[t]{2}{*}{ Galates } & $\varepsilon \gamma \varrho \alpha \varphi \eta \alpha \pi \circ$ P $\omega \mu \eta \varsigma$ & $\begin{array}{l}\text { B }^{1} \text { K L P } 42698117391881 \\
1908 \text { Byz; } c d^{1} \text {, vg }\end{array}$ \\
\hline & écrite d'Ephèse & $\operatorname{vg}^{\mathrm{R}}$ \\
\hline Ephésiens & $\varepsilon \gamma \varrho \alpha \varphi \eta \alpha \pi \circ$ P $\omega \mu \eta \varsigma$ & $\begin{array}{l}\text { B }^{1} \text { K L P } 698232817391881 \\
1908 \text { Byz; c d } d^{1} \text { vg.R }^{\mathrm{L} . \mathrm{R}}\end{array}$ \\
\hline \multirow[t]{2}{*}{ Philippiens } & $\varepsilon \gamma \varrho \alpha \varphi \eta \alpha \pi \circ$ P $\omega \mu \eta \varsigma$ & $\begin{array}{l}\text { B }^{1} \text { K L } 66917391881 \text { Byz; } c \\
d^{1} \mathrm{vg}^{\text {H.Lmg.R.T.U.Z. } \Theta}\end{array}$ \\
\hline & $\varepsilon \gamma \varrho \alpha \varphi \eta \varepsilon \xi \mathrm{A} \theta \eta \nu \omega \nu^{7}$ & 945 \\
\hline Colossiens & $\varepsilon \gamma \varrho \alpha \varphi \eta(-\mathrm{A}) \alpha \pi \mathrm{P} \mathrm{P} \omega \mu \eta \varsigma$ & 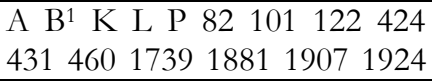 \\
\hline
\end{tabular}

2 NeSTLE-AlAND, Novum Testamentum graece, 27e éd., Stuttgart, 1993.

3 J. WOrdwOrTH - H.J. WhITE - H.F.D. SPARKS, Novum Testamentum Domini nostri Iesu Christi latine, vol. 2, Oxford, 1941.

${ }^{4}$ Nestle indique seulement $1881 p c$.

${ }^{5}$ Ephèse est le lieu indiqué dans la préface de l'éd. euthalienne, d'après Tischendorf.

${ }^{6} \mathrm{D}^{\mathrm{abs} 1}$ désigne le Sangermanensis, ancien manuscrit Ep, qui est une copie du Claromontanus (D).

${ }^{7}$ La provenance d'Athènes est inconnue de l'apparat de Tischendorf. 


\begin{tabular}{|c|c|c|}
\hline & & Byz; $c d^{1} \mathrm{vg}^{\text {H.Lmg.R.T.U. } \Theta}$ \\
\hline \multirow[t]{4}{*}{1 Thess } & $\varepsilon \gamma \varrho \alpha \varphi \eta \alpha \pi \sigma$ A $\theta \eta \nu \omega \nu$ & $\begin{array}{l}\text { A } \text { B }^{1} \text { K L L } 17391881 \text { Вуг; } \\
\text { vg }\end{array}$ \\
\hline & 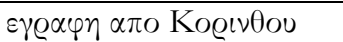 & $81 p c$; Euthalius \\
\hline & écrite de Laodicée & $d^{1}$ \\
\hline & écrite de Rome & $e$ (en début d'épître) \\
\hline \multirow[t]{2}{*}{2 Thes } & $\varepsilon \gamma \varrho \alpha \varphi \eta \alpha \pi 0 \mathrm{~A} \theta \eta \nu \omega \nu^{8}$ & $\begin{array}{l}\text { A B }{ }^{1} \text { K L P } 81 \text { 1739txt Byz; } d^{1} \\
\text { vg }\end{array}$ \\
\hline & $\varepsilon \gamma \varrho \alpha \varphi \eta \alpha \pi \circ$ P $\omega \mu \eta \varsigma$ & 63284526141739 mg 1912 \\
\hline \multirow[t]{3}{*}{ Hébreux $^{9}$} & $\varepsilon \gamma \varrho \alpha \varphi \eta \alpha \pi o$ ( $\tau \eta \varsigma) I \tau \alpha \lambda \iota \alpha \varsigma$ & $\begin{array}{l}\text { K P }(0243)^{10} 0285101102404 \\
4254314604641908 \text { Byz; vg }\end{array}$ \\
\hline & $\varepsilon \gamma \varrho \alpha \varphi \eta \alpha \pi \circ$ P $\omega \mu \eta \varsigma$ & A \\
\hline & $\varepsilon \gamma \varrho \alpha \varphi \eta \alpha \pi \sigma$ A $\theta \eta \nu \omega \nu$ & 1911 (par erreur ?) \\
\hline \multirow[t]{2}{*}{1 Timothée } & $\varepsilon \gamma \varrho \alpha \varphi \eta \alpha \pi \sigma \Lambda \alpha O \delta ı \varepsilon \iota \alpha \varsigma$ & $\begin{array}{l}\text { A K L } 241173918811908 \\
\text { Byz; } c d^{1} v^{\text {H.Lmg. T.U. } \Theta}\end{array}$ \\
\hline & 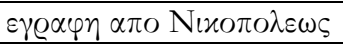 & P 102 \\
\hline \multirow[t]{2}{*}{2 Timothée } & $\varepsilon \gamma \varrho \alpha \varphi \eta \alpha \pi \sigma$ P $\omega \mu \eta \varsigma$ & $\begin{array}{l}\text { K P } 646417391881 \text { Byz; } d^{1} e \text {, } \\
\text { vgLmg }\end{array}$ \\
\hline & 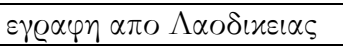 & A ; vg H.T.U. $\Theta$ \\
\hline \multirow[t]{2}{*}{ Tite } & 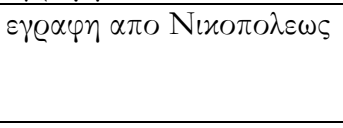 & $\begin{array}{l}\text { A H K L P } 8110117391881 \\
19081927 \text { Byz; c d e, vg } \\
\text { vg.Lmg. }\end{array}$ \\
\hline & $\varepsilon \gamma \varrho \alpha \varphi \eta \alpha \pi o$ M $\alpha x \varepsilon \delta o v i \alpha \varsigma$ & 945 \\
\hline Philémon & $\varepsilon \gamma \varrho \alpha \varphi \eta \alpha \pi \circ$ P $\omega \mu \eta \varsigma$ & $\begin{array}{l}\text { K L P } 0484269101241390 \\
4621739188119081927 \text { Byz; } \\
c d^{1} e, \text { vg }\end{array}$ \\
\hline
\end{tabular}

Ajoutons quelques précisions sur les manuscrits cités.

\section{Codex Vaticanus (B.03)}

Bible grecque copiée vers 340 (C. del Vaticano, BAV, Vat. Gr. 1209), principal témoin du texte alexandrin de tout le Nouveau Testament ${ }^{11}$; mais les souscriptions sont postérieures à la copie ( $7^{\mathrm{e}} \mathrm{s}$. ?). Le Vaticanus est précédé, dans la tradition alexandrine, par le Papyrus de Chester Beatty II (P46), qui n'a pas de souscription; il est contemporain du Sinaïticus (\$.01) qui n'en présente pas ; et le Codex de Freer II (I.016) n'en a pas non plus. Aucun autre témoin alexandrin ne présente de souscription : celles-ci ne viennent donc pas de la tradition alexandrine. $\mathrm{La}$ fin du corpus, après $\mathrm{Hb} 9,14$, est lacuneuse : la fin d'Hébreux (sans souscription) et l'Apocalypse ont été suppléées au $15^{\mathrm{e}} \mathrm{s}$., mais il manque les Pastorales et Philémon.

\section{Codex Alexandrinus (A.02)}

Bible grecque copiée au $5^{\mathrm{e}} \mathrm{s}$. (London, BL, Royal V-VIII), avec les indications de lieu en souscription pour certaines épîtres seulement (de Colossiens à Tite), de première main : c'est le premier témoin des souscriptions des épîtres de Paul. Dans ce manuscrit, le texte des évangiles est byzantin, avec des variantes alexandrines; le reste du Nouveau Testament présente un texte alexandrin ; mais les souscriptions appartiennent à une autre tradition.

\footnotetext{
${ }^{8}$ Le Vaticanus (B) est lacuneux pour la fin d’Hébreux et les épîtres suivantes.

${ }^{9}$ L'Angelicus (L) est lacuneux pour la fin d'Hébreux.

10 L'oncial 0243 donne l'indication de lieu en inscription (au début d'Hébreux).

11 P. ANDrist (éd.), Le manuscrit B de la Bible (Vat. Gr. 1209) (Histoire du texte biblique 7), Lausanne, 2009.
} 


\section{Fragments 048 (palimpseste, texte inférieur)}

Il reste de ce manuscrit fragmentaire du $5^{\mathrm{e}} \mathrm{s}$. (type de texte mixte) quelques feuillets des Actes et des épîtres de Paul qui ont été effacées puis incluses parmi d'autres feuillets ${ }^{12}$ (C. del Vaticano, Vat. Gr. 2061). La souscription de Philémon confirme que celles-ci sont ajoutées dès le $5^{\mathrm{e}} \mathrm{s}$..

\section{Codex Claromontanus (D.06/d75)}

Manuscrit bilingue grec-latin des épîtres de Paul (Paris, BnF, gr. 107), copié au $\sigma^{\mathrm{e}}$ s. sur un modèle proche de deux autres bilingues, l'Augiensis (F.010/f.78) et le Boernerianus (G.012/g.77) et ayant servi de modèle au Sangermanensis (Dabs1/e.76), tous trois copiés au $9^{\mathrm{e}} \mathrm{s}$. Ces manuscrits sont les témoins du texte «occidental» des épîtres de Paul : le corpus comprend les Pastorales, mais pas Hébreux. Les souscriptions sont ajoutées après la copie dans le Claromontanus, du côté grec pour les deux premières lettres seulement. Hébreux figure en annexe dans le manuscrit, séparé du corpus par une liste latine de livres bibliques et sans souscription.

\section{Codex Coislinianus (H.015)}

Manuscrit fragmentaire des épîtres de Paul copié au $6^{\mathrm{e}}$ s., dont il reste 41 feuillets dispersés dans huit bibliothèques ${ }^{13}$, ayant un texte alexandrin, une seule souscription (Tite) et la division euthalienne des épîtres. Dans un colophon, le copiste signale que son modèle vient de la bibliothèque de Pamphile, à Césarée. On a donc un témoin d'une édition euthalienne avec texte alexandrin et une souscription.

\section{Codex fragmentaire 0285}

L'oncial 081, dont il reste 2 feuillets devenus des pages de garde dans un manuscrit de St Pétersbourg (GPB, Gr. 9), est désormais l'oncial $0285^{14}$, dont 20 autres feuillets ont été retrouvés dans le nouveau fonds du monastère Ste Catherine du Sinaï (MГ 70) : ce sont des fragments d'un codex du $6^{\mathrm{e}}$ s. contenant les épittres du NT ; il reste une seule souscription, celle d'Hébreux.

\section{Codex Mosquensis (K.018), Codex Angelicus (L.020), Codex Porphyrianus (P.025, palimpseste, texte inf.)}

Trois onciaux du $99^{\mathrm{e}} \mathrm{s}$. contenant les Actes et les épîtres et ayant des souscriptions à la fin des épîtres de Paul ${ }^{15}$. Le modèle de ces manuscrits n'est pas le même : $\mathrm{L}$ est le plus byzantin, $\mathrm{K}$ et $\mathrm{P}$ ont des variantes qui les distinguent du texte byzantin sans les rattacher aux types plus anciens. Tous trois présentent des lacunes.

\section{Codex en onciales 0278 (palimpseste, texte supérieur, grec-arabe)}

Ce manuscrit bilingue grec-arabe des épîtres de Paul, copié au 9e s., dont il reste 120 feuillets avec des souscriptions (il en subsiste quatre), a été retrouvé dans le nouveau fonds du monastère Ste Catherine du Sinaï (MГ 2), copié sur un écrit théologique effacé ${ }^{16}$. Le type de texte n'est pas connu.

\section{Codex fragmentaire 0243}

Il reste 9 feuillets (dont 7 à Venise et 2 à Hambourg) de ce recueil des épîtres de Paul $^{17}$ copié au $10^{\mathrm{e}}$ s., avec souscriptions à la fin des lettres.

12 C.R. Gregory, Textkritik des NT, vol. 1, Leipzig, 1900, p. 104 ; K. ALAND, Kurzgefasste Liste der griechischen Handschriften des NT, 2e éd., Berlin New York, 1994, p. 23.

13 Gregory, op. cit., p. 114-116; AlAND, op. cit, p. 20. Le texte a été rassemblé et édité par H. OMONT, Notice sur un très ancien manuscrit grec en onciales des épîtres de saint Paul conservé à la Bibliothèque nationale, Paris 1890.

14 ALAnd, op. cit, p. 43.

15 GREGORY, op. cit., p. 101-102 ; ALAND, op. cit, p. 20-22.

16 Aland, op. cit, p. 42.

17 GREgORY, op. cit., p. 117-118 ; ALAND, op. cit, p. 39. 


\section{Codex en semi-onciales 075 (commentaire en minuscules)}

Manuscrit des épîtres de Paul avec commentaire ${ }^{18}$, copié au $10^{\mathrm{e}}$ s., pourrait être classé parmi les minuscules, avec lesquels il offre une transition. Le manuscrit a encore quelques souscriptions, il est conservé à Athènes (BE, 100).

\section{Les minuscules}

Les éditions de Tischendorf et de Nestle-Aland citent, par ailleurs, une cinquantaine de minuscules, copiés du $10^{\mathrm{e}}$ au $16^{\mathrm{e}} \mathrm{s}$, , choisis plus pour la particularité de leurs souscriptions que pour leur représentativité parmi plusieurs centaines de manuscrits; en voici un aperçu encore plus bref que pour les onciaux :

1) 8 sont copiés au 10e s. (82 338450602605173919121927$), 14$ au $11^{\mathrm{e}}$ (42 8110110424125242446694519071908192319241932$), 8$ au $12^{\mathrm{e}}$ (122 242337431436452462 618), 8 au 13e (6 328339390404460469 614), 4 au 14e (209 425603 1881), 3 au 15e (69 102 205) et 2 au 16e (90 1911); leur contenu varie: p (1907(K) 1908(K) 19111912 1923(K) $1924(\mathrm{~K}) 1927(\mathrm{~K}) 1932(\mathrm{~K})$, dont plusieurs avec commentaire $(\mathrm{K}))$; ap $(\mathbf{8 1}$ 101(K) 102328404425436450460 (g-l-arb) 462466602603 605(K) 614 6181739 1881) ; apr (42 82(K) 104337 424(K) 452 469); eap (6 90122 252390431 945) ; eapr (69 209241242 339) ; et le 205 est le NT d'une bible grecque.

2) Les manuscrits en gras ont pour le corpus paulinien des variantes anciennes pré-byzantines, mais tous ont subi l'influence du texte byzantin; les souscriptions font dans la plupart des cas partie de cette influence.

3) Liste des manuscrits cités (dans l'ordre du répertoire) : 642698182 90101102104122205209241242252328337339390404424425 43143645045246046246646960260360561461894517391881 19071908191119121923192419271932

Les minuscules illustrent la diversité de la tradition byzantine, avec quelques variantes sur le lieu de copie; dans l'ensemble, ils confirment l'information donnée par les premiers onciaux, déjà confirmée par les onciaux tardifs.

\section{Vieille latine}

L'indication de lieu ne figure pas dans la Vieille latine, mais elle est ajoutée de seconde main dans $d$, recopiée dans $e$ (d'après $d$ ) et dans $c$ elle est de tradition vulgate.

$c$ : codex Colbertinus, $13^{\mathrm{e}}$ s., conservé à Paris $(\mathrm{BnF}$, lat. 254$)$; texte vulgate

pour Paul ;

$d:$ latin du codex Claromontanus (voir grec);

$e$ : latin du codex Sangermanensis, copie du Claromontanus (voir grec) ;

\section{Vulgate latine}

Quelques manuscrits seulement attestent les indications de lieu dans les souscriptions, notamment l'édition de Théodulfe $\left(9^{\mathrm{e}} \mathrm{s}\right.$.) et la branche représentée par l'Harleianus.

G : codex Sangermanensis (bible latine), $9^{\mathrm{e}}$ s., conservé à Paris (BnF, lat. 11553);

$\mathrm{H}$ : codex Hubertianus, 8 $8^{\mathrm{e}} 9^{\mathrm{e}}$ s., conservé à Londres (BL, Add. 24142)

$\mathrm{L}$ : codex Langobardus, $8^{\mathrm{e}}$ s., conservé à Paris (BnF, lat. 335) ;

$\mathrm{R}$ : codex Reginensis, ${ }^{\mathrm{e}}$ s., conservé au Vatican (BAV, Reg. Lat. 9) ;

$\mathrm{T}$ : codex Toletanus, $10^{\mathrm{e}}$ s., conservé à Madrid (BN, Vitr. 4 / Tol. 2. 1) ;

$\mathrm{U}$ : codex Ulmensis, ${ }^{\mathrm{e}}$ s., conservé à Londres (BL, Add. 11852) ;

$\mathrm{Z}$ : codex Harleianus, 6e s., conservé à Londres (BL, Harl. 1775);

$\Theta$ : codex Thedulfianus, 9e s., conservé à Paris (BnF, lat. 9380).

\footnotetext{
18 GregOrY, op. cit., p. 309 [min. 382] ; AlAND, op. cit, p. 27.
} 
Au total, 9 lettres présentent une variation du lieu de leur rédaction :

$\begin{array}{ll}1 \text { Corinthiens : } & \text { Ephèse ou Phi } \\ \text { 2 Corinthiens : } & \text { Philippes (M } \\ \text { Galates : } & \text { Rome ou Eph } \\ \text { Philippiens : } & \text { Rome ou A } \\ 1 \text { Thessaloniciens : } & \text { Athènes ou } \\ 2 \text { Thessaloniciens : } & \text { Athènes ou } \\ \text { Hébreux : } & \text { Italie (Rome) } \\ 1 \text { Timothée : } & \text { Laodicée (P } \\ 2 \text { Timothée : } & \text { Rome ou La } \\ \text { Le lieu de rédaction est consensue } & \\ & \text { Corinthe ; } \\ \text { Romains : } & \text { Rome ; } \\ \text { Ephésiens : } & \text { Rome ; } \\ \text { Colossiens : } & \text { Nicopolis ; } \\ \text { Tite : } & \text { Rome. }\end{array}$

En bref, les indications de lieux de rédaction des épîtres de Paul figurent, en grec comme en latin, dans les souscriptions, par introduction tardive dans une partie de la tradition manuscrite. Leur importance parait donc limitée ; pourtant, à l'examen, nous allons voir qu'elles ne sont pas sans intérêt, mais que celui-ci est inégal et parfois indirect.

\section{L'interprétation des souscriptions}

\section{Le rappel des voyages de Paul}

Pour évaluer l'intérêt des lieux de rédactions des épîtres de Paul, il faut avoir en mémoire le parcours missionnaire de Paul, tel qu'il figure dans les Actes des apôtres ${ }^{19}$.

Un premier voyage de Paul a lieu avant la conférence de Jérusalem de 49 (Ac 15) et se déroule en Anatolie centrale (Ac 13-14) : aucune des villes où prêche Paul n'est mentionnée comme lieu de rédaction, ce voyage n'entre donc pas en compte comme le temps où Paul écrit ses lettres.

Les deux voyages de Paul autour de la Mer Egée (Ac 16-18 et 19-20) séparés par un retour de Paul à Antioche (Ac 18,22), précédés et suivis d'une étape à Jérusalem, ont un grand intérêt : Paul participe à la conférence de Jérusalem en 49 , en qualité de représentant de l'Eglise d'Antioche; et au printemps 58, il est arrêté à Jérusa-

${ }^{19}$ Les données existantes ne suffisent pas pour établir une chronologie de la vie de Paul. Voir les introductions au NT, mais aussi, par exemple : M.-F. BASLEZ, Saint Paul, aritsan d'un monde chrétien, Paris, 1991, p. 441-445; et S. LEgAsse, Paul apôtre, Paris - Saint Laurent (Québec), 2000², p. 13-24. Nous nous en sommes tenu à un choix plus traditionnel, fixant la conversion de Paul en 35, quatorze ans avant la conférence de Jérusalem en 49 , son séjour à Ephèse de 55 à 57 , puis son arrestation à Jérusalem au printemps 58 ; et son séjour à Rome, de 61 à 63. 
lem, au terme de son deuxième voyage égéen (Ac 21). Les étapes de ces deux voyages correspondent à plusieurs lieux indiqués dans les souscriptions.

Une fois en prison, à Jérusalem, puis à Césarée (Ac 21-26), Paul comparait en justice et fait appel, ce qui lui vaut un transfert à Rome. Son voyage se fait alors par mer, il est l'occasion d'une tempête (Ac 27-28), et Paul arrive finalement à Rome, pour un séjour que les Actes ne racontent pas, le livre s'arrêtant sur l'arrivée de Paul. Cette ultime étape romaine correspond également à un des lieux invoqués pour la rédaction des lettres du corpus paulinien.

Au cours de son premier voyage égéen, Paul gagne l'Anatolie depuis Jérusalem par la Syrie et la Cilicie, puis il traverse différentes régions jusqu'à Troas, d'où il gagne la Macédoine et s'arrête à Philippes, où il baptise Lydie (Ac 16,11-15). Paul se rend ensuite à Thessalonique, où il rencontre l'hostilité de la communauté juive et poursuit sa route jusqu'à Athènes (Ac 17,16-34) et se rend à Corinthe, capitale romaine de la Grèce, où il passe dixhuit mois; puis il retourne par mer à Antioche, en passant par Ephèse (Ac 18,18-22).

Le deuxième voyage part d'Antioche, Paul traverse à nouveau l'Anatolie et arrive à Ephèse, capitale de l'Asie romaine, où il passe deux ans (Ac 19,10). Puis il se rend en Grèce (= à Corinthe) en passant par la Macédoine (Ac 20,1-3), revient par terre à Philippes et gagne Troas où, au cours d'une fraction du pain, un jeune homme meurt et ressuscite (Ac 20,7-12) ; Paul s'embarque alors pour Milet après plusieurs brèves escales (Assos, Mitylène, Chios, Ac 20,13-15), et il y retrouve les anciens d'Ephèse ; puis il s'embarque pour Césarée, d'où il gagne à Jérusalem.

En somme, en deux voyages, Paul fait étape principalement dans dix villes distinctes, parfois plusieurs fois ; il séjourne plus d'un an dans deux des capitales régionales, Corinthe ( $1^{\text {er }}$ voyage) et Ephèse ( $2^{\mathrm{e}}$ voyage).

\section{Les lettres dans le ministère de Paul}

L'exégèse des lettres du corpus paulinien attribue à Paul une partie seulement de ces lettres : pour la plupart des exégètes, cinq d'entre elles sont écrites par un disciple, il s'agit d'Ephésiens, des Pastorales (1-2 Timothée, Tite) et d'Hébreux. Deux autres lettres sont également suspectes d'être l'œuvre d'un héritier de Paul, 2 Thessaloniciens et Colossiens. Il reste, en somme, sept lettres sur quatorze qui seraient l'œuvre authentique de Paul : Romains, 1-2 Corinthiens, Galates, Philippiens, 1 Thessaloniciens et Philémon. Voyons donc d'abord, pour les lettres sûrement de Paul, si les lieux de rédaction indiqués dans les souscriptions sont fiables.

Romains se présente comme écrite à Corinthe, où Paul séjourne longuement lors de son premier voyage égéen, puis reste trois mois, lors du deuxième voyage. Le lieu indiqué est fiable. 
L'exégèse a admis que cette épittre majeure avait été écrite lors du séjour de Paul « en Grèce » (Ac 20,3), soit dans l'hiver 57-58 ${ }^{20}$.

1 Corinthiens serait écrite soit à Ephèse, soit à Philippes, les témoins sont partagés. Or, Paul séjourne longuement à Ephèse lors de son deuxième voyage égéen ; plus brièvement à Philippes, d'abord au début de son premier voyage - c'est là qu'il rencontre et baptise Lydie -, puis au cours du second, après Ephèse, en se rendant en Grèce (passant par la Macédoine) et en en revenant, pour gagner Troas et l'Anatolie. D'après son contenu, la lettre est écrite peu de temps avant celle aux Romains, donc soit pendant le séjour à Ephèse (Ac 19), soit juste après, quand Paul passe par la Macédoine pour aller en Grèce (Ac 20,1-2) ; autrement dit, entre 55 et 57 . Mais les lettres suivantes vont permettre d'être plus précis.

2 Corinthiens serait écrite plutôt à Philippes, peut-être à Troas : la rédaction à Philippes au moment où Paul se rend en Grèce, lors de son deuxième voyage égéen, est la solution la plus probable, admise par l'exégèse ; mais le court passage de Paul rend peu vraisemblable qu'il ait écrit aussi 1 Corinthiens, soit deux longues lettres au même moment à la même communauté. En somme, 1 Corinthiens date du séjour à Ephèse, ce qui rend mieux compte de l'insistance de Paul sur Apollos, en début de lettre (1 Co 1-4). 2 Corinthiens daterait ainsi de 57.

Galates serait écrite depuis Rome, d'après la plupart des témoins, ou à Ephèse, selon un seul témoin latin. Rome ne convient pas pour cette lettre, tandis que le début du séjour à Ephèse irait très bien : Paul s'oppose à un faux évangile qu'il vient de découvrir en arrivant à Ephèse, et il met en garde ses correspondants. La lettre daterait en réalité de 55 et serait antérieure à 1 Corinthiens, écrite dans ces conditions en 56. La localisation à Rome de Galates doit donc recevoir une autre explication.

Philippiens serait écrite plutôt à Rome ou encore à Athènes, mais aucune de ces deux villes ne s'accorde avec le contenu, qui correspond à une période de captivité ; le plus probable est que Paul a écrit cette lettre, en une ou plusieurs fois, à Césarée, en attente d'un transfert vers Rome, donc vers 59. Il faut donc aussi rendre compte du lieu de rédaction indiqué.

1 Thessaloniciens serait écrite à Athènes ou Corinthe; des témoins latins ajoutent deux autres lieux moins crédibles; Laodicée ou Rome. La lettre est considérée comme la toute première de Paul, car elle contient une allusion au retour de Jésus avant la fin de la génération (4,13-17), idée à laquelle Paul renonce ensuite et qui est présente dans la prédication des apôtres de Jérusalem, d'après plusieurs témoignages. Cet accord entre Paul et les apôtres correspond à son premier voyage égéen, au cours duquel il passe par Thessalonique, puis se rend à Athènes avant un long séjour à Corinthe. Il est donc probable que la lettre a bien été écrite soit à

\footnotetext{
${ }^{20}$ Voir par exemple la note de la TOB à Actes 20,3.
} 
Athènes, soit au début du séjour à Corinthe, au cours du premier voyage égéen de Paul, vers l'an 50.

Philémon, enfin, est attribuée à Paul, ce billet serait écrit depuis Rome et correspond par son contenu à la période où Paul s'y trouve, avec une nouvelle mention de Marc et Luc (v. 25), soit entre 61 et 63.

Envisageons à présent les deux lettres dont l'attribution à Paul est encore souvent admise.

2 Thessaloniciens serait écrite, selon les témoins, à Athènes ou à Rome ; mais aucun de deux lieux ne convient: Paul ne peut pas écrire deux lettres aux mêmes destinataires lors de son court passage à Athènes, en développant des idées différentes; et le contenu de la lettre ne correspond pas à sa période romaine. L'attribution à Paul est donc contestée, comme celle de Colossiens; pourtant, cette lettre correspond à une étape de la vie de Paul, celle dont il fait état dans Galates ${ }^{21}$, en relatant la division des tables, à Antioche, entre Judéo- et pagano-chrétiens. Le constat ne peut avoir lieu qu'au moment où Paul revient à Antioche, à l'issue de son premier voyage égéen, et cet «incident» est sans doute l'une des causes de son départ pour un deuxième voyage autour de la Mer Egée. On peut donc envisager l'authenticité de cette lettre en l'associant au passage de Paul à Antioche, vers 54 ; mais il reste à expliquer la fausse indication de lieu qui lui est donnée.

Colossiens serait écrite à Rome, d'après tous les témoins ; un doute subsiste sur son auteur, mais nous verrons qu'un argument documentaire va dans le sens de l'authenticité de l'épitre ; et le contenu de la lettre correspond bien à la période romaine de Paul, avec la mention de Marc et Luc comme ses continuateurs $(4,10-$ 14), soit entre 61 et 63 .

Passons à présent aux lettres dont l'attribution à Paul est peu vraisemblable.

Ephésiens n'est pas de Paul pour plusieurs raisons : d'abord, elle fait état d'une organisation de la communauté qui correspond à la génération d'après Paul ; ensuite, elle suit un schéma qui est un décalque de Colossiens ${ }^{22}$. Cette similitude entre les deux épîtres suffit à expliquer que le lieu de rédaction de la deuxième soit repris à la première. Mais quelle est la réalité ? Ephésiens fait partie d'un groupe de lettres qui ont d'abord servi à accompagner un écrit évangélique et qui ont ensuite été diversement intégrés au

\footnotetext{
${ }^{21}$ Galates 2,11-14. Paul met en cause la responsabilité de Pierre et Jacques dans cette division.

22 On lit, par exemple, dans l'introduction de la TOB à Ephésiens : «On constate, à propos d'Ephésiens, que tous les détails ayant trait aux données historiques sont presque littéralement empruntés à Colossiens. De plus, l'apôtre ne connait pas personnellement les destinataires $(1,15):$ il ne peut donc s'agir de l'Eglise d'Ephèse, où Paul a fait un séjour prolongé (...) La relation Ephésiens/Colossiens constitue une des énigmes du Nouveau Testament. Cette énigme n'a pas encore trouvé d'explication satisfaisante ».
} 
Nouveau Testament; et dans ce cadre, elle est l'œuvre probable d'Aristion, écrivant depuis Smyrne à l'Eglise d'Ephèse vers 80 : Papias lui attribue des "Récits des paroles du Seigneur", autrement dit une rédaction évangélique ${ }^{23}$. Le chef de l'Eglise d'Ephèse est alors Jean l'apôtre, assisté d'un autre Jean dit «le Presbytre», autre maître de Papias, qui lui attribue des «traditions» particulières ${ }^{24}$, soit une part probable à la rédaction de l'évangile selon Jean.

Hébreux fait partie du même groupe de lettres qu'Ephésiens : elle intègre une collection de citations de l'Ancien Testament appliquées à Jésus qui a probablement existé avant la lettre et pouvait être à Rome au temps où Paul s'y trouve, ce qui peut expliquer le lieu de rédaction indiqué ; et un détail au début du chap. 10, permet de dater la lettre d'avant 70: selon Clément d'Alexandrie, elle serait de Luc et aurait servi à accompagner son premier livre ${ }^{25}$.

1 Timothée serait écrite à Laodicée ou Nicopolis, 2 Timothée, à Rome ou Laodicée, et Tite, à Nicopolis : en dehors de Rome pour 2 Timothée, qui reste une hypothèse possible, les autres lieux ne correspondent pas aux étapes des voyages de Paul, ce qui renforce l'opinion que ce sont des lettres d'un autre auteur, attribuée à Paul lors de leur intégration au corpus.

Au total, nous avons situé neuf des quatorze lettres du corpus paulinien dans la chronologie du ministère de Paul, dans l'ordre suivant :

1 Thessaloniciens, écrite à Athènes ou Corinthe, vers l'an 50 (premier voyage égéen);

2 Thessaloniciens, écrite à Antioche [et non Athènes ou Rome], vers l'an 54 (entre les deux voyages égéens) ;

Galates, écrite au début du séjour à Ephèse [et non Rome], en 55 ou 56

(deuxième voyage égéen) ;

1 Corinthiens, écrite au cours du séjour à Ephèse, en 56 ou 57 (deuxième voyage égéen) ;

2 Corinthiens, écrite à Philippes, en 57 (deuxième voyage égéen) ;

Romains, écrite à Corinthe, hiver 57-58 (deuxième voyage égéen) ;

Philippiens, écrite en prison à Césarée [et non Athènes ou Rome], vers 59

(captivité de Paul) ;

Colossiens, écrite à Rome, entre 61 et 63 (fin du ministère de Paul ;

Philémon, écrite à Rome, entre 61 et 63 (fin du ministère de Paul).

Pourquoi certains lieux sont-ils exacts et d'autres, fantaisistes ?

Pour les cinq autres lettres, écrites vraisemblablement après Paul, on doit rendre compte de leur attribution à Paul (sauf Hébreux) et du lieu indiqué comme celui de la rédaction.

${ }^{23}$ Eusèbe de Césarée, Histoire ecclésiastique 3, 39,14. Voir C.-B. AmpHOux, «La grande lacune du Codex de Bèze », Filologia neotestamentaria 17 (2004), p. 325, spécialement p. 15.

${ }^{24}$ Voir Eusèbe, même passage que n. 22.

${ }^{25}$ Art. cit. (n. 23), spécialement p. 15. 


\section{La constitution du corpus paulinien}

C'est au cours de son second voyage égéen, entre 55 et 58 , que Paul écrit les lettres dont la théologie va devenir celle des chrétiens, en particulier 1 Corinthiens et Romains. Mais la pensée de Paul n'est pas immédiatement reçue : il faut attendre la fin des années 60 , puis la destruction du temple en 70 , pour que son influence marque la rédaction de nouveaux écrits qui serviront de sources à la rédaction finale des évangiles. On a supposé que certaines lettres de Paul étaient alors déjà rassemblées ${ }^{26}$. Mais aucune trace de tels corpus n'existe avant le début du $2^{\mathrm{e}} \mathrm{s}$.

Clément de Rome, dans sa lettre écrite depuis Rome aux Corinthiens vers 95, est le premier à citer explicitement une lettre de Paul : «Reprenez la lettre du bienheureux apôtre Paul : que vous a-t-il d'abord écrit, au commencement de l'évangile ${ }^{27}$ ? $(47,1-2)$; puis il rappelle ce que Paul dit à propos de "Céphas et Apollos ${ }^{28}$ " (47,3 : cf. 1 Co 1-4) et l'hymne à l'amour (49 : cf. 1 Co 13). Antérieurement, il a évoqué la mort rédemptrice («le sang du Christ... versé pour notre salut», 7,4) et repris la phrase de Gn 15,6 «Abraham eut foi en Dieu et elle lui fut comptée comme justice » $(10,6)$, commentée par Paul (Rm 4-7), pour substituer la foi à la loi comme chemin de salut. Clément connait, en somme, au moins ces deux lettres de Paul et rien n'indique encore qu'elles soient réunies en un corpus avec d'autres lettres. Mais on peut noter que, pour ces deux lettres, les lieux de rédaction indiqués en souscription sont fiables : ce sont deux lettres datant du deuxième voyage égéen de Paul, 1 Corinthiens étant écrite avant Romains.

Ignace d'Antioche, dans sa lettre aux Ephésiens écrite peu avant les autres, vers 110 , groupe cinq allusions à des lettres pauliniennes (chap. 8-10): (1) le mot "râclure » $(8,1)$ est repris de 1 Corinthiens 4,13; (2) le couple «charnels / spirituels» $(8,2)$

${ }^{26}$ D. Trobisch, Die Entstehung der Paulusbriefsammlung. Studien zu den Anfängen christlicher Publizistike (Novum Testamentum et Orbis Antiquus 10), Göttingen, 1989 / The First Edition of the NT, Oxford, 2000, considère que Paul a le premier réuni quatre de ses lettres, Romains, 1-2 Corinthiens et Galates. F. Vouga signale cette hypothèse et envisage la constitution de deux collections «après la mort de l'apôtre » (voir D. MARGUERAT (éd.), Introduction au NT, 2e éd., Genève, 2001, p. 150). Mais ces conjectures de premières collections n'ont pas d'appui documentaire. Prudemment, R. E. BROWN (Que sait-on du NT?, Paris, 2000) élude la question de la réunion des lettres pauliniennes.

${ }^{27}$ L'expression apparaît en $\mathrm{Phl}$ 4,15 pour renvoyer au début du ministère de Paul (premier voyage égéen); elle est aussi une reprise du titre d'une source évangélique "Commencement de l'évangile de Jésus Christ», qui deviendra le début de Marc $(1,1)$.

${ }^{28} \mathrm{Il}$ est admis que Céphas soit une manière de désigner Pierre comme représentant des apôtres ; ce qui l'est moins c'est l'identité d'Apollos, dont il est clair qu'il représente le groupe des Hellénistes; à cause d'Apollonios, variante « occidentale » d'Apollos en Ac 18,24, c'est-à-dire Apolloni(os), le deuxième élément étant l'anagramme du nom de Jean (Oni- / Ioan-), il faut se demander, en complément de la savante étude de P.F. BEATRICE ( Apollos of Alexandria and the Origins of the Jewish-Christian Baptist Encratism »dans Aufstieg und Niedergang der Römischen Welt II.26.2, Berlin - New York, 1995, p. 1232-1275), si Apollos ne désigne pas l'apôtre Jean, qui disparait de la direction de la communauté primitive après le départ des Hellénistes (Ac 8). 
reprend Romains 15,27 ; (3) «les pierres du temple préparées pour la construction» $(9,1)$ reprennent l'idée de construction, développée en Ephésiens 2,19-22 ; (4) "priez sans cesse » $(10,1)$ est une reprise de 1 Thessaloniciens 5,17; enfin, (5) «soyez fermes dans la foi » $(10,2)$ reprend Colossiens 1,23. La lettre d'Ignace présente d'autres groupements d'allusion qui se prêtent à une lecture métonymique: par la disposition que l'on observe dans chaque groupe, Ignace indique comment réunir les écrits qui constituent, de son point de vue, la "tradition des apôtres ", dont Eusèbe garde la mémoire qu'il jugeait essentiel de la fixer par écrit $^{29}$. Ainsi, en quelques mots, par une savante métonymie, Ignace suggère de constituer un corpus de cinq lettres, dans le cadre d'un projet littéraire plus vaste, qui sont, dans l'ordre :

1 Corinthiens - Romains - Ephésiens - 1 Thessaloniciens - Colossiens

Le corpus des lettres de Paul est alors simplement suggéré, il devra comprendre cinq lettres: (1) deux lettres longues (1 Corinthiens, Romains), déjà citées par Clément de Rome, qui contiennent la part essentielle de la pensée de Paul ; (2) une lettre centrale (Ephésiens), qui n'est pas de Paul, mais qui a son autorité et est déjà connue des destinataires de la lettre d'Ignace ; (3) deux lettres courtes (1 Thessaloniciens, Colossiens), l'une datant du début du ministère de Paul et l'autre, de la période finale. La présence de Colossiens dans ce premier groupe d'épîtres constitue un argument documentaire pour considérer cette épître comme étant bien de Paul. Or, les quatre lettres qui prennent ainsi place dans le ministère de Paul ont en souscription un lieu de rédaction fiable ; et pour la lettre centrale, le lieu indiqué est celui de Colossiens, qui lui a servi de modèle. On voit apparaître une première raison de la disparité des lieux de rédaction indiqués : certains ont un fondement historique probable, d'autres reposent sur une analogie.

Dans ses lettres suivantes, Ignace fait encore allusion à 2 Corinthiens et Philippiens, deux lettres qui suggèrent un élargissement du corpus en projet, sans indiquer de place aux nouvelles venues, mais amplifiant le premier noyau d'une lettre longue et d'une courte; en respectant la structure précédente, cela donne : 3 lettres longues -1 lettre centrale -3 lettres courtes, soit :

1 Corinthiens - 2 Corintbiens - Romains/Ephésiens/1 Thes - Colossiens - Philippiens

L'indication de lieu des deux lettres est analogique de celle qui les précède : 1 Corinthiens, d'un côté, Colossiens, de l'autre ; pour la première, l'indication de lieu est historiquement fiable, les deux lettres aux Corinthiens semblent bien écrites au cours du deu-

${ }^{29}$ «Et tandis qu'il voyageait à travers l'Asie, il affermissait les Eglises... et les pressait de tenir fermement à la tradition des apôtres que, pour plus de sécurité, il estimait nécessaire de fixer encore par écrit » (Eusèbe, Hist. eccl. 3, 36,4). Un jeu sur les noms propres permet d'associer «Burrhus » à la tradition d'Antioche et "Onésime» à celle d'Ephèse. Voir D. Rouger - C.-B. AMPHOUX, «Le projet littéraire d'Ignace d'Antioche dans son épitre aux Ephésiens », Mél. J.K. Elliott, à paraître en 2013. 
xième voyage égéen de Paul, lors du séjour à Philippes ou un peu avant; pour la seconde, en revanche, l'analogie masque la réalité, qui est probablement le rassemblement de plusieurs billets écrits par Paul quand il est en prison à Césarée. Les deux lettres ont un rapport avec Philippes, qui est peut-être le lieu où ces lettres ont été gardées avant d'être portées à la connaissance d'Ignace, toujours vers 110 . La localisation de la rédaction par analogie s'étend donc à ces deux nouvelles lettres.

Polycarpe de Smyrne est le continuateur d'Ignace et le probable réalisateur du corpus qu'il suggère, en cinq ou sept lettres; mais une telle édition ne nous est pas parvenue ; or, comme nous allons le voir, Polycarpe pourrait être à l'origine du rassemblement des dix lettres du corpus de Marcion.

Marcion, à Rome vers 140, publie le premier corpus attesté des lettres de Paul, qui comprend dix lettres, et précède de peu la rupture de l'éditeur avec l'Eglise romaine et la naissance de sa propre Eglise. Le corpus de Marcion, reconstitué par A. von Harnack $^{30}$, est le suivant :

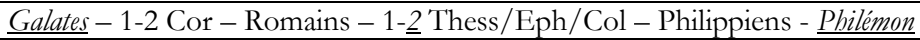

On retrouve dans ce corpus les sept lettres que nous venons de voir, avec les allusions d'Ignace, et trois nouvelles lettres, Galates, 2 Thessaloniciens et Philémon. L'ensemble est disposé de manière à former autour d'Ephésiens, désormais adressée «aux Laodicéens », une proposition remarquable qui s'inspire de celle des cinq lettres au groupement suggéré par Ignace. Au lieu de deux lettres longues et de deux plus courtes, de part et d'autre d'une lettre centrale, on a une proportion du simple au double inversée, par le nombre de lettres et celui des communautés destinataires :

6 lettres à 4 communautés / 1 lettre centrale / 3 lettres à 2 communautés

Or, Marcion n'est pas partisan de cette rhétorique savante, qui groupe les écrits d'une manière significative et que l'on observe dans le texte « occidental» du Nouveau Testament. Marcion est à la recherche d'un langage simple, il révise en particulier l'évangile de Luc dans ce sens, il n'est donc pas l'inventeur de cette disposition, mais il reprend probablement une proportion établie avant lui, qu'il est le premier à attester et qu'il a pu aménager à sa façon.

La proportion du simple au double inversée vient compléter une double proportion que l'on peut observer dans la bible grecque, comprenant la proportion d'égalité et la proportion du simple au double, qui a pour origine une spéculation de la philosophie grecque: entre le monde de Dieu et celui qui est matériel, Dieu a institué un lien, une «âme », et lui a donné la structure de l'octave, c'est-à-

\footnotetext{
30 A. vOn Harnack, Marcion. Das Evangelium vom fremden Gott, Beilage III : «Das Apostolikon Marcions », Leipzig, $1924^{2}$, p. 40*-176*.
} 
dire la double proportion en question ${ }^{31}$. Les nombres qui lient la quarte et la quinte à l'octave, découverts par Pythagore, réalisent cette double proportion qui forme, dans l'école pythagoricienne, la structure de l'octave. Autrement dit, la double proportion signifie par sa présence un lien entre Dieu et le monde terrestre ; autrement dit, elle donne aux livres qu'elle lie le statut d'Ecriture sacrée. Et la proportion du simple au double inversée apparaît dans la bible grecque chrétienne en complément de la double proportion, pour ajouter à ce statut l'idée de réconciliation avec Dieu, attachée à la venue du messie. Ainsi, Daniel se trouve ajouté à la fin du corpus des Prophètes ${ }^{32}$.

Le corpus de Marcion serait, en somme, d'abord constitué par Polycarpe, l'éditeur probable d'un double corpus, à Smyrne, vers 120-130 : celui des quatre évangiles dans l'ordre Matthieu - Jean Luc - Marc, formant autour de la Femme adultère (introduite dans Jean en 7,53-8,11) une double proportion : d'égalité, pour les paroles de Jésus, et $d u$ simple au double, pour les parties narratives ${ }^{33}$; et celui des lettres paunliniennes, disposées dans la proportion $d u$ simple au double inversée, pour compléter la double proportion précédente $^{34}$.

L'indication du lieu de rédaction est clairement analogique pour 2 Thessaloniciens, qui serait écrite à Athènes, selon la tradition byzantine, qui situe également à Athènes 1 Thessaloniciens ; il en est de même pour Philémon, qui serait écrite à Rome comme Colossiens, avec laquelle elle a en commun la mention de Marc et Luc parmi les continuateurs de Paul ; pour la première, l'analogie masque le lieu, qui est plutôt Antioche qu'Athènes (voir plus haut); alors que le billet à Philémon est probablement bien écrit à Rome. Mais pour Galates, l'analogie ne suffit pas à expliquer l'indication de Rome comme lieu de rédaction qui n'a pas de fondement historique ; il faut donc trouver une autre explication. Le corpus de Marcion place en tête les quatre lettres du deuxième voyage égéen dans leur ordre chronologique probable; et les trois lettres qui suivent Galates ont une localisation fiable : pourquoi n'en est-il pas de même pour Galates? Doit-on admettre une relation entre la position de Galates en tête du corpus publié par Marcion et le fait que cette édition se fasse à Rome ? Dans cette

31 Voir C.-B. Amphoux, «L'âme du monde du Timée de Platon : une composante de la rhétorique biblique », dans A. BALANSARD - G. DORIVAL - M. LOUBET (éd.), Prolongements et renouvellements de la tradition classique, Mél. D. Pralon, t. 2 (Textes et documents de la Méditerranée antique et médiévale), Aixen-Provence, 2011, p. 121-132.

${ }^{32}$ Les Prophètes ont une disposition nouvelle : les XII - Esaïe - Jérémie Baruch - Lamentations - Lettre de Jr - Ezéchiel - Daniel ; les XII font le double de la longueur de Daniel grec (avec ses amplifications); Esaïe et Jérémie font ensemble deux fois la longueur d'Ezéchiel; et Baruch a deux fois la longueur de la Lettre de Jérémie, de part et d'autre du livret central des Lamentations. Telle est, en particulier, la disposition attestée dans le Codex Vaticanus (B), après la double proportion observable dans les livres précédents.

33 Voir C.-B. Amphoux, «La place de l'épisode de la Femme adultère (Jn 7,53-8,11)», Mél. J. Pelaez, à paraître.

${ }^{34}$ Voir art. cit. [note 23]. 
éventualité, le lieu de rédaction serait introduit par Marcion pour signifier que son édition s'est faite à Rome; et ce lieu se serait transmis comme étant celui de la rédaction de Galates.

Deux passages du texte «occidental» permettent de faire l'hypothèse que le lieu transmis pour la rédaction de Galates est en réalité celui de l'édition de Marcion : en Mt 2,11, les présents des mages à Jésus ont un deuxième sens, ils indiquent le cheminement de l'écriture de l'évangile qu'ils commencent, et $\sigma \mu \dot{\varrho} \varrho v \alpha$, la "myrrhe », est aussi le nom de la ville de Smyrne, où est publiée l'édition des évangiles, avec l'ordre et le texte "occidental», par Polycarpe; de même, en Ac 2,9, la présence de l'article devant "Asie », dans une liste où les autres régions sont sans article, équivaut à un possessif, "notre Asie », indiquant ainsi que c'est dans cette région que l'édition qui inclut ce livre a été faite, autrement dit plus précisément à Smyrne par Polycarpe. Le procédé de « signer » une édition n'est donc pas inconnu, Marcion le reprend sans doute à la génération qui le précède. Au total, la chronologie des lettres n'est pas parfaitement respectée, mais le corpus de Marcion place, avant la lettre centrale, les lettres écrites au cours des deux voyages égéens, et après elle, celles qui suivent l'arrestation à Jérusalem; pour une chronologie parfaite, il faudrait pour cela que le corpus commence par 1-2 Thessaloniciens et que Philippiens précède Colossiens. Un tel corpus a-t-il été réuni avant lui et réorganisé pour placer en tête les lettres dont il partageait la théologie, à commencer par Galates? On peut en faire l'hypothèse.

Quoi qu'il en soit, les lettres les plus anciennement rassemblées ont des indications de lieu historiquement fiables, puis celles des lettres ajoutées seraient analogiques, enfin la première du corpus publié par Marcion porterait le lieu de son édition et non de la rédaction de la lettre. Une logique lie ainsi les lieux de rédaction transmis et l'histoire de la formation du corpus.

Le Codex Claromontanus et trois autres bilingues grecslatins plus tardifs ${ }^{35}$ présentent un deuxième groupement des lettres de Paul, avec 13 lettres, dont les Pastorales, mais pas Hébreux :

$$
\text { Ro-1-2 Co-Gal / Eph / Col-Phl-1-2 Th-1-2 Tm-Tt-Phm }
$$

Dans ce corpus élargi, la disposition demeure organisée autour de l'épître aux Ephésiens (qui a retrouvé ses premiers destinataires) : quatre lettres la précèdent, où Romains, la principale de toutes les lettres, se trouve désormais en tête, suivie d'une lettre double et de Galates ; et huit lettres la suivent, avec deux doubles au milieu : la volonté de l'éditeur est de disposer les lettres dans la proportion du simple au double (et non plus la proportion du simple au double inversée). Le sens du corpus a donc changé : le corpus antérieur, réalisé vers 120-130 et repris par Marcion, s'expliquait

${ }^{35}$ Les codex Augiensis, Boernerianus et Sangermanensis sont du $9^{\mathrm{e}}$ s., voir plus haut. 
comme le complément de celui des évangiles, dans la logique du projet d'Ignace ; le corpus amplifié s'explique, quant à lui, comme le complément de celui du Codex de Bèze, qui ajoute à la double proportion des évangiles (voir plus haut) une proportion du simple au double inversée, constituée par les quatre évangiles, d'un côté, et deux livres (Actes, Apocalypse), de l'autre, de part et d'autre d'un groupe de lettres, lui-même formant une proportion du simple au double inversée ${ }^{36}$. Ce groupement peut être daté vers 160, il correspond à une édition de la fin de la vie de Polycarpe ${ }^{37}$, et il forme, avec un premier corpus contenant les évangiles et d'autres livres du Nouveau Testament, un groupement de 26 des 27 écrits néotestamentaires, il ne manque que Jude. Dans le corpus paulinien amplifié, les lettres déjà réunies changent de disposition, mais la tradition de leur lieu de rédaction se transmet, y compris pour Galates ; et un témoin latin atteste que le probable lieu de rédaction ne s'est pas complètement oublié.

Il reste à rendre compte des lieux invoqués pour la rédaction des nouvelles lettres : ces lieux (Laodicée, Nicopolis) ne se trouvent pas sur le parcours des voyages de Paul ; mais ce sont peutêtre les lieux où elles ont bien été écrites; cette fois, c'est probablement le véritable auteur qui est masqué, par l'attribution à Paul; et le lieu indiqué est historiquement probable. Ajoutons, pour 2 Timothée, qu'une partie de la tradition dit écrite à Rome, que cette mention pourrait correspondre à un billet ou une lettre de Paul qui serait une première rédaction de la lettre que nous lisons désormais.

Le Papyrus de Chester Beatty II $\left(\mathrm{P}^{46}\right)$, copié vers 200, atteste un troisième groupement que l'on peut dater d'après le Canon de Muratori ${ }^{38}$, autrement dit après 180 : il comprend le corpus de Marcion avec Hébreux après Romains, mais pas les Pastorales, et les lettres sont réorganisées dans le sens décroissant de leur longueur :

Ro - He - 1-2 Co - Gal - Eph - Col - Phl - 1 Th... [2 Th - Phm]

Dans ce manuscrit, il manque les sept feuillets extrêmes du début (Ro 1-5) et de la fin: ceux-ci comprenaient la fin de 1 Thessaloniciens, puis la place suffisante pour 2 Thessaloniciens et Philémon, mais pas pour les Pastorales (1-2 Timothée - Tite). Ce groupement a une probable origine alexandrine ${ }^{39}$, à tout le

${ }^{36}$ Voir art. cit. [note 23].

${ }^{37}$ La lettre de Polycarpe, jointe à celles d'Ignace dans le corpus des Pères apostoliques, présente un groupement de citations d'écrits du Nouveau Testament qui correspond à ce stade : les Pastorales sont citées au milieu des épîtres de Paul, et Hébreux l'est juste avant les épîtres catholiques, auxquelles elle est probablement associée.

38 Rédigé vers 180, le Canon de Muratori atteste les lettres de Paul avec Hébreux et sans les Pastorales. Sur la date du Canon, voir C.-B. AmPHOUX, «Le canon du NT avant le 4e siècle », Filologia neotestamentaria 21 (2008), p. 9-26.

${ }^{39}$ Eusèbe (Hist. eccl. 5, 10,4) prête à Pantène un rôle actif à la tête de l'école chrétienne d'Alexandrie : ce rôle pourrait comprendre une édition des évangiles vers 175, avant le Canon de Muratori et avant le traité de Celse sur le «Véri- 
moins égyptienne. Il associe pour la première fois Hébreux au corpus paulinien, alors que celle-ci n'est pas explicitement attribuée à Paul, mais elle contient un ensemble de citations qui était peut-être déjà constitué du temps de Paul. Hébreux est connue de Clément de Rome, comme d'autres lettres non pauliniennes, en particulier 1 Pierre. Et l'on peut faire l'hypothèse que le lieu de rédaction généralement indiqué, l'Italie, est plutôt celui de la collection de citations appliquées à Jésus que celui de toute la lettre.

Le Codex Sinaïticus et le Codex Vaticanus (‥01, B.03), deux bibles grecques copiées entre le Concile de Nicée (325) et 350, sont les premiers témoins du corpus complet des lettres de Paul qui s'impose en grec, à partir du $4^{\mathrm{e}} \mathrm{s}$. : il comprend au total quatorze lettres, avec Hébreux avant les Pastorales, Philémon fermant ainsi le corpus :

Ro-1-2 Co - Gal-Eph - Phl - Col-1-2 Th- He-1-2 Tm-Tt-Phm

Cet ordre est aussi celui des bibles grecques du $5^{\mathrm{e}}$ s. (A.02, C.04). Il faut attendre le $9^{\mathrm{e}}$, pour que l'ordre actuel avec Hébreux en fin de corpus apparaisse, en concurrence avec l'ordre du $4^{\mathrm{e}} \mathrm{s}$.

\section{Conclusion}

Les lieux de rédaction figurant dans les souscriptions des lettres de Paul, sur les manuscrits grecs et latins, fournissent des données concernant l'histoire de la formation du corpus; et tous sont, à ce titre, dignes d'intérêt.

Les quatre premières lettres dont le groupement est suggéré dans la lettre d'Ignace d'Antioche aux Ephésiens, écrite vers 110, sont 1 Corinthiens, Romains, 1 Thessaloniciens et Colossiens : elles ont un lieu de rédaction historiquement fiable, qui concorde avec leur date probable: 1 Corinthiens, à Ephèse (plutôt que Philippes), écrite vers 57, et Romains, à Corinthe, fin 57 à début 58, au cours du deuxième voyage égéen de Paul ; 1 Thessaloniciens, à Athènes, vers 50, au cours du premier voyage égéen; et Colossiens, à Rome, entre 61 et 63, à la fin de la vie de Paul.

La lettre centrale suggérée par Ignace pour la réunion de ces lettres est Ephésiens, qui a les mêmes destinataires que la lettre d'Ignace ; elle est l'œuvre d'un disciple de Paul qui utilise Colossiens pour modèle : le lieu de rédaction indiqué (Rome) est analogique de celui de Colossiens ; en réalité, selon mon hypothèse, ce

table Logos »; et une édition du corpus paulinien, peu après 180, suivie par une édition du psautier qui en subit l'influence : voir C.-B. AMPHOUX - G. DORIVAL, «'Des oreilles, tu m'as creusées' ou 'Un corps, tu m'as ajusté' ? A propos du Ps 39 (40 TM), 7 », dans P. Brillet-Dubois - E. Parmentier (éds.), Philologia, Mél. M. Cazevitz (Collection M.O.M. 35, série littéraire et philosophique 9), Lyon, 2006, p. 315-327. 
serait l'œuvre d'Aristion, prédécesseur de Polycarpe, et elle serait écrite à Smyrne, vers 80-90.

Deux nouvelles lettres apparaissent dans les autres lettres d'Ignace, toujours écrites vers 110 , et leur lieu de rédaction indiqué s'explique encore par l'analogie : 2 Corinthiens serait écrite à Philippes, lieu attribué à 1 Corinthiens; et Philippiens, à Rome, d'après Colossiens. Pour la première, le lieu indiqué est historiquement fiable, la lettre daterait de 57 ; pour la seconde, le lieu probable est au contraire masqué, la lettre serait écrite vers 59, à Césarée, peut-être en plusieurs fois.

Trois nouvelles lettres apparaissent dans le corpus de Marcion: pour 2 Thessaloniciens et Philémon, c'est encore par l'analogie qu'on peut expliquer les lieux indiqués; pour la première, l'analogie avec 1 Thessaloniciens masque le lieu probable, qui est Antioche, soit une lettre écrite entre les deux voyages égéens, vers 54 ; pour Philémon, le lieu est historiquement fiable, car le contenu concorde avec Colossiens, la rédaction se situe donc à Rome, entre 61 et 63. Pour la troisième lettre, Galates, l'analogie ne suffit pas à expliquer que sa rédaction soit localisée à Rome, car elle prend place en début de corpus : ni historique ni analogique, ce lieu est l'héritage probable de celui de l'édition des lettres de Paul par Marcion. On conserve des indications de lieu comparables dans le texte « occidental » des évangiles et des Actes.

Les lettres pastorales, 1-2 Timothée et Tite, entrent dans le corpus seulement vers 160 et ne s'y fixent pas encore définitivement : les lieux de rédaction sortent du trajet missionnaire de Paul et ont peut-être un rapport avec leur véritable auteur, plus tardif que Paul, mais non identifié ; les villes de Laodicée et Nicopolis sont donc peut-être des lieux historiques de rédaction; quant à l'indication de Rome parfois donnée pour 2 Timothée, elle est soit une analogie, soit la trace d'une première rédaction faite à Rome par Paul.

L'épitre aux Hébreux, enfin, rejoint un corpus sans les Pastorales vers 180, à Alexandrie, et prend place juste après Romains : la lettre ne porte pas le nom de Paul dans son adresse, elle est clairement l'œuvre d'un paulinien; et le lieu indiqué, non pas une ville, mais un pays, l'Italie, pourrait s'appliquer à la constitution du florilège de citations de la bible juive que contient la lettre, plutôt qu'à la rédaction finale de l'épître : selon mon hypothèse, celle-ci serait l'œuvre de Luc, écrivant à la fin des années 60, plutôt à Antioche qu'en Italie.

On voit, par ces valeurs différenciées du lieu figurant dans les souscriptions des lettres du corpus paulinien, que chaque mention de lieu a son intérêt, car toutes sont en rapport avec l'histoire de la constitution du corpus des épîtres de Paul. Ce sont des données documentaires qui corrigent l'idée reçue que le corpus est constitué dès la fin du $1^{\text {er }} \mathrm{s}$. et qui sont importantes à ce titre. 